\title{
Menopausa precoce no Brasil: uma revisão bibliográfica integrativa
}

\author{
Early menopause in Brazill: an integrative bibliographical revision \\ La menopausia temprana en Brasil: una revisión bibliográfica integrativa
}

\author{
Mônica de Mendonça Mustafa \\ ORCID: https://orcid.org/0000-0002-6499-6666 \\ Universidade Nilton Lins, Brasil \\ E-mail: monicamustafa@outlook.com \\ Edna Paula P. de Souza \\ ORCID: https://orcid.org/0000-0002-7324-7480 \\ Universidade Nilton Lins, Brasil \\ E-mail: edna_paula1@hotmail.com \\ Alysson Bastos Sena \\ ORCID: https://orcid.org/0000-0002-0562-8433 \\ Universidade Nilton Lins, Brasil \\ E-mail: alyssonsena@hotmail.com
}

\begin{abstract}
Resumo
A menopausa precoce é uma condição que afeta $1 \%$ das mulheres, consistindo na a cessação permanente da menstruação por perda de função ovariana antes dos 40 anos. Este artigo analisa quantitativamente a bibliografia que cita o termo em quatro indicadores diferentes: a) existe disparidade entre homens e mulheres nas pesquisas sobre o tema? b) Quais as regiões das instituições que os pesquisadores em questão estão vinculados? c) Quais as especialidades dos autores que citam a menopausa precoce? d) A menopausa precoce é tratada como foco das pesquisas ou não? É feita então uma análise qualitativa sobre o que esses artigos falam dividindo-os por categorias de abordagem semelhante: os que relacionam a menopausa precoce à osteoporose; os que relacionam-na a câncer; os que relacionam a mesma à menopausa genericamente; artigos que citam-na mas não tem coesão interna ou volume suficiente para uma categoria exclusiva. $\mathrm{O}$ artigo conclui que uma quantidade maior de mulheres são autoras em artigos que citaram o termo, que o sudeste e nordeste concentram a maior parte de autores, estando a presença de pesquisadores da região norte abaixo das demais, que apesar de muitas áreas do conhecimento estarem presentes, a medicina é a de maior peso e que a menopausa precoce não é tratada como foco principal em nenhum dos artigos encontrados; essas questões mostram que há espaço para a expansão de estudos sobre a menopausa precoce no Brasil, tanto ao estuda-la enquanto fenômeno biológico quanto estudando as políticas públicas de saúde e pesquisa sobre a mesma.
\end{abstract}

Palavras-chave: Menopausa precoce; Menopausa; Saúde pública; Saúde da mulher.

\begin{abstract}
Early menopause is a condition that affects $1 \%$ of women, consisting in the permanent cessation of menstruation due to the loss of ovarian function before the age of 40 years. This article does a quantitatively analysis of the bibliography that cites the term in four different indicators: a) is there a disparity between men and women in research on the subject? b) In what regions are the institutions of the authors of the selected papers? c) What are the areas of knowledge of the authors who cite early menopause? d) Is early menopause treated as the focus of the paper or not? A qualitative analysis is then made by categorizing the main themes of the article's relation to the early menopause: those that relate early menopause to osteoporosis; those that relate it to cancer; those that relate it to menopause generically; articles that cite it but do not have enough internal cohesion or volume for an exclusive category. It is concluded that more women are authors in articles that cited the term; that the Southeast and Northeast concentrate the majority of authors, with the presence of researchers from the northern region being lower than the others; that despite the fact that many areas of knowledge are present, medicine is the one with the heaviest weight; that early menopause is not treated as the main focus in any of the articles found. These questions show that there is room for the expansion of studies on early menopause in Brazil, both when studying it as a biological phenomenon or public health policies and research on it.
\end{abstract}

Keywords: Early menopause; Menopause; Public healthcare; Women's healthcare.

\section{Resumen}

La menopausia precoz es una condición que afecta al $1 \%$ de las mujeres y consiste en el cese definitivo de la menstruación debido a la pérdida de la función ovárica antes de los 40 años. Este artículo hace un análisis cuantitativo de la bibliografía que cita el término en cuatro indicadores diferentes: a) ¿Existe disparidad entre hombres y mujeres en la investigación sobre el tema? b) ¿En qué regiones se encuentran las instituciones de los autores de los artículos seleccionados? c) ¿Cuáles son las áreas de conocimiento de los autores que citan la menopausia precoz? d) ¿Se trata la 
menopausia precoz como el tema central del artículo o no? A continuación, se realiza un análisis cualitativo categorizando los temas principales de la relación del artículo con la menopausia precoz: los que relatan la menopausia precoz con la osteoporosis; los que lo reportan al cáncer; los que lo reportan a la menopausia de forma genérica; artículos que lo citan pero que no tienen suficiente cohesión interna o volumen para una categoría exclusiva. Se concluye que más mujeres son autoras en los artículos citados con el término; que el Sudeste y Nordeste concentran la mayoría de los autores, siendo menor la presencia de investigadores de la región norte que los demás; que a pesar de que existen muchas áreas de conocimiento, la medicina es la de mayor peso; que la menopausia precoz no se trata como el foco principal en ninguno de los artículos encontrados. Estas preguntas muestran que hay espacio para la expansión de los estudios sobre la menopausia precoz en Brasil, tanto al estudiarla como fenómeno biológico como a las políticas de salud pública y la investigación sobre ella.

Palabras clave: Menopausia precoz; Menopausia; Atención de la salud pública; Salud de la mujer.

\section{Introdução}

Segundo a Organização Mundial da Saúde a menopausa é "a cessação permanente da menstruação por perda de função ovariana" e a "menopausa natural acontece depois de 12 meses consecutivos de ausência de menstruação sem causas patológicas" (Lay, 2018, p.11).

A partir do climatério, período em que a mulher se encontra na transição entre fase reprodutiva e não reprodutiva, ocorrem irregularidades constantes no ciclo menstrual. O corpo deixa de secretar estrogênio na mesma quantidade que fez durante o período de pleno funcionamento e cria um ambiente biológico hipoestrogênico com consequente aumento na produção de androgênios e tendência ao aumento dos níveis de FSH produzidos pela hipófise (Antunes et al, 2003).

Todas essas transformações ocasionam mudanças físicas e psicológicas na mulher: sentem maior dificuldade em adormecer, alterações de humor, atrofia da mucosa vaginal, cefaleia, fogachos, palpitações, irritação e depressão (Santos et al, 2016).

Segundo Lay (2018), a média brasileira da menopausa é de 51 anos, embora possa acontecer também em diferentes momentos da vida da mulher. A OMS preconiza que a menopausa ocorra entre os 51 e 55 anos ocorrendo três variações importantes além da considerada natural. A menopausa considerada precoce ocorre antes dos 40 anos, a tardia após os 53 anos e a artificial ocorre após ooforectomia (retirada de um ou dos dois ovários). (Correa \& Casagrande, 2021). Esse estudo propõe identificar o tratamento dado ao fenômeno da menopausa precoce na literatura entre 2016-2021.

$\mathrm{O}$ artigo conclui que uma quantidade maior de mulheres são autoras em artigos que citaram o termo, que o sudeste e nordeste concentram a maior parte de autores, estando a presença de pesquisadores da região norte abaixo das demais, que apesar de muitas áreas do conhecimento estarem presentes, a medicina é a de maior peso e que a menopausa precoce não é tratada como foco principal em nenhum dos artigos encontrados; essas questões mostram que há espaço para a expansão de estudos sobre a menopausa precoce no Brasil, tanto ao estuda-la enquanto fenômeno biológico quanto estudando as políticas públicas de saúde e pesquisa sobre a mesma.

\section{Metodologia}

A metodologia utilizada é a revisão bibliográfica integrativa que permite analisar estudos experimentais ou não, combinando literaturas empíricas e teóricas para discutir os fenômenos em sua complexidade real (Sousa et al, 2010), buscando pela palavra-chave "menopausa precoce" nos repositórios google acadêmico, scientific eletronic library online (SCIELO), pubmed e scopus, analisando artigos que citem necessariamente esse termo, estejam em português e tenham sido publicados em periódicos acadêmicos no período entre 2016-2021. Sendo o fenômeno de interesse a menopausa precoce e não havendo outro termo que designe o fenômeno, a busca teve foco apenas na presença dessa palavra-chave.

Os textos fornecerão dados para responder as seguintes perguntas, permitindo cumprir com os objetivos da pesquisa: a) quais os efeitos da menopausa precoce na saúde da mulher? b) qual o gênero dos autores desses artigos: masculino ou feminino? c) qual a região dos departamentos aos quais estão vinculados: norte, nordeste, centro-oeste, sudeste ou sul? d) os autores 
escrevem a partir de qual disciplina: medicina, farmácia, enfermagem, psicologia, etc.? e) quais são as abordagens que são expostas no texto sobre a menopausa precoce: tema central de trabalhos ou periféricos, como dados secundários ou exemplos? Foram encontrados inicialmente 43 textos dentro desses parâmetros, mas 9 foram falsos-positivo por na verdade terem sido publicados fora do recorte, tendo apenas sido adicionados aos repositórios online nesse período. Com isso, a seleção final foi de 34 artigos.

Figura 1. Artigos selecionados para a pesquisa.

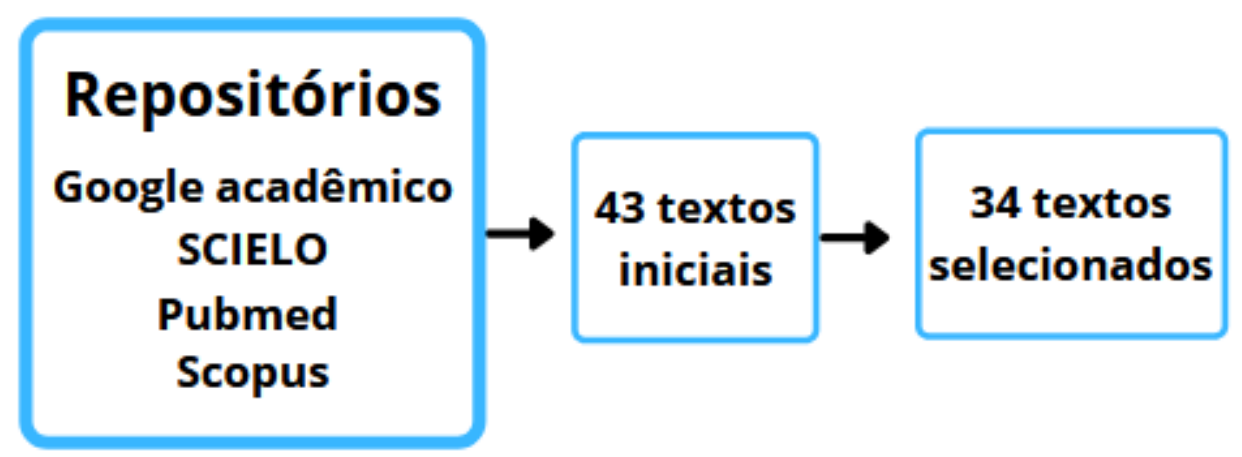

Fonte: Autores.

Após esse movimento, é feita uma análise qualitativa sobre o que esses artigos falam dividindo-os por categorias de abordagem semelhante: aqueles que relacionam a menopausa precoce à osteoporose; os que relacionam-na a diferentes tipos de câncer; os que relacionam a mesma ao fenômeno da menopausa genericamente; artigos que citam-na mas não tem coesão interna ou volume suficiente para uma categoria exclusiva.

\section{Discussão e Resultados}

Como exposto por Oliveira et al (2012), há uma percepção de que certas doenças e condições, por serem raras, só são pesquisadas e têm medidas implementadas a partir da mobilização em massa de pessoas que se envolvem comunitariamente por medidas relativas a ela, indo da conscientização até chegar às políticas públicas.

Por mais que a menopausa precoce não seja uma patologia, ela é uma condição que pode trazer desdobramentos patológicos como aumento da probabilidade de osteoporose (Alves et al, 2021) e os efeitos negativos à qualidade de vida citados anteriormente, embora afete apenas por volta de $1 \%$ das mulheres.

Este estudo se propôs a fazer uma revisão bibliográfica integrativa sobre a menopausa precoce, analisando como ela é tratada na literatura, o que auxiliará pesquisadores em futuros esforços de pesquisa e na criação de políticas públicas de acordo com as regiões em que se produzem os artigos analisados, áreas de atuação dos pesquisadores e qual o volume de pesquisas que citam o tema.

Foi feita uma revisão de artigos científicos publicados no Brasil entre 2016-2021, apresentando as perspectivas apresentadas no conhecimento científico para esse fenômeno. Inicialmente serão expostos os dados quantitativos sobre a produção analisada: número de artigos publicados por ano, gênero dos autores, região da instituição a que estão vinculados, área 
de formação e o destaque dado ao tema como central ou não no trabalho. No segundo momento será feita uma discussão qualitativa sobre quais os dados que envolvem a menopausa precoce presentes nos textos analisados.

$\mathrm{Na}$ busca pela palavra-chave "menopausa precoce" nos repositórios acadêmicos google, scholar, pubmed, scielo e scopus foram encontrados 34 artigos publicados em periódicos acadêmicos entre 2016-2021 em português que citaram o termo. Nestes artigos há 35 autores homens e 118 autoras mulheres. A prevalência de mulheres enquanto autoras e coautoras da literatura é absolutamente clara. Esse dado está em consonância com a caracterização das tendências sobre a força de trabalho da saúde no Brasil, como o texto de Wermelinger et al (2010), sendo possível fazer uma inferência hipotética inicial de que nossa amostra se comporta aproximadamente como os dados estatísticos exprimem a caracterização do campo: enquanto o Wermelinger et al (2010) apresentam a razão de $31 \%$ homens e $69 \%$ mulheres na força de trabalho no Brasil, o gráfico produzido pelo levantamento feito para este artigo mostra a razão aproximada de $23 \%$ homens e $77 \%$ mulheres.

Após esses dados, o levantamento sobre a região dos autores foi feito com base em suas vinculações institucionais de formação básica embora, quando em conflito, a titulação mais alta fosse representativa: um pós-graduando que já integra algum corpo docente e atua profissionalmente em região diferente de onde está concluindo sua formação, portanto, é alocado à região onde trabalha. Isso se justifica pelo fato de que a pós-graduação é uma ocupação com vínculo temporário e intencionalmente deve terminar em algum momento, ao contrário do posto de trabalho que possui algum nível de perenidade em sua natureza. Foram encontrados os seguintes dados: 3 autores da região norte; 53 do nordeste; 19 centro-oeste; 53 do sudeste; 17 do sul.

O termo "menopausa precoce" é citado majoritariamente em artigos publicados por autores das regiões sudeste e nordeste, seguidos pelo centro-oeste e sul, e por último, com pouca representação, a região norte. É difícil inferir se há alguma motivação específica para esse fato: ao hipotetizar que poderia haver alguma correlação com a incidência de menopausa precoce de acordo com as regiões não é possível fazer uma primeira inferência que sustente a hipótese e permita trabalha-la mais profundamente pela falta de dados, embora seja um indicativo interessante para que seja feito um estudo com maior profundidade.

Outra hipótese que poderia ser levantada, por sua vez, é a de que há no nordeste e sudeste mais pesquisadores da área da saúde. Isso, entretanto, não explicaria tão grande diferença entre essas duas regiões e a região sul, também com uma grande quantidade de centros universitários e de pesquisa, embora entender esse fenômeno pudesse revelar alguma especificidade sobre a população daquela região: há nela menor incidência de menopausa precoce, fazendo com que haja pouca produção sobre a mesma? A incidência é a mesma da média nacional? Se sim, por que a disparidade na quantidade de estudos é tão alta? São muitas questões possíveis, todas de alta importância para políticas públicas que envolvem pesquisa na área da saúde.

Além disso, a pequena representação de citações no norte poderia ser atribuída a um menor número de universidades e centros de pesquisa, o que nos leva à duas informações: tanto a região centro-oeste quando a região norte possuem 11 universidades públicas federais e nenhuma das duas regiões tem diferenças expressivas em universidades estaduais; essa diferença talvez pudesse se encontrar nos programas de universidades privadas, mas voltamos à dificuldade de obtenção dos dados, o que não permite dar substância à hipótese. Esse, contudo, é um problema que se estudado poderia auxiliar na melhor alocação de recursos públicos em educação e pesquisa relativas à saúde, fortalecendo o desenvolvimento de pesquisas sobre saúde na região norte.

Foram levantados dados também sobre as especialidades dos autores que publicaram os artigos, o que permitiu gerar as seguintes informações: 49 - medicina; 15 - enfermagem; 6 - farmácia; 6 - fisioterapia; 5 - biomedicina; 5 - educação física; 4 - odontologia; 3 - biologia; 3 - nutrição; 1 - biotecnologia. 
Esses dados, entretanto, são apenas uma aproximação, uma vez que muitos dos autores não informaram tanto em seus artigos quanto em seus currículos, majoritariamente ORCIDs $^{1}$, qual sua área de formação e atuação, impossibilitando a inclusão integral dos mesmos na base de dados. Isso não impede, por exemplo, que seja possível ver forte predominância de profissionais da medicina citando a menopausa precoce, seguidos pela enfermagem. Todos os demais profissionais que citam o termo aparecem reduzidamente nos artigos analisados. Esses dados apresentam um cenário que, apesar de abordado por diversos profissionais, tem grande ênfase nos conhecimentos médicos. Talvez isso explique em partes porque grande parte da literatura analisada nesse artigo cita a menopausa precoce apenas como fator de risco ou consequência de outras patologias e seus tratamentos. Há poucas citações relacionadas ao tratamento de reposição hormonal e métodos para amenizar sintomatologias da menopausa, abordagens interessantes para estudos que poderiam ser enriquecedoras nesse campo, que poderiam vir das demais disciplinas como farmácia, biomedicina, biotecnologia e biologia, por exemplo. Conclui-se, portanto, que há espaço para crescimento da colaboração interdisciplinar em relação aos estudos da menopausa precoce, especificamente.

Por último, a série histórica de artigos publicados onde o termo "menopausa precoce" é citado, mostrando a distribuição cronológica no período de 2016-2021 é de 5 citações em 2016, 4 em 2017, 3 em 2018, 6 em 2019, 11 em 2020, 5 em 2021. Apesar do claro pico de citações no ano de 2011, é difícil trazer alguma hipótese dada a variedade temática dos trabalhos que o fazem, indo da correlação entre menopausa precoce e osteoporose até a prática sexual de mulheres jovens com câncer de mama.

Entretanto, é possível que uma série histórica mais ampla possa abrir novas leituras desse dado mostrando se ele está ou não dentro de uma variação normal: se sim, o dado mostra que há uma constância no volume de pesquisas sobre o tema que não foi impactada pelo crescimento da estrutura de pesquisa no Brasil desde o início dos anos 2000; se não, ela aumentou ou diminuiu? É importante ter esses dados claros para ver quando a menopausa precoce passou a ser estudada no Brasil e se a tendência é que cada vez mais estudos falem da mesma ou que ela seja abordada apenas lateralmente na bibliografia, indicando necessidade ou não de uma postura ativa do Estado para aumentar estudos sobre o assunto.

O último dado disponível a partir da análise quantitativa sobre o conjunto dos artigos é o de que, dos 34 artigos em foco, nenhum dá ênfase à menopausa precoce. Em todos a mesma aparece em relação a algo, nunca em destaque, e a melhor forma de avaliar como isso ocorre é analisando esses artigos qualitativamente. Os conteúdos, por sua vez, podem ser divididos da seguinte forma em quatro categorias relativas à menopausa precoce: câncer; osteoporose; menopausa; outros.

Os artigos que citam menopausa precoce e tratam sobre o câncer são Pereira et al (2017), Silva Oliveira et al (2018), Lovison et al (2019), Mairink et al (2020), Barros de Oliveira et al (2021) e Corrêa et al (2017).

Os textos de Pereira et al (2017), Lovison et al (2019), Mairink et al (2020), Barros de Oliveira (2021) e Corrêa et al (2017) citam a menopausa precoce como um dos possíveis impactos do tratamento para o câncer de mama, sendo essa a abordagem predominante. Silva Oliveira et al (2018), entretanto, usa a menopausa precoce como uma variável para a análise da propensão ao câncer de mama, apresentando uma abordagem fora da tendência geral e tendo como resultado que das mulheres que desenvolveram câncer de mama na população de sua pesquisa, apenas $5 \%$ tiveram menopausa precoce, apontando a hipótese de que a correlação parece ser fraca ou nula.

Já Carvalho et al (2020), Fidelis et al (2020), Alves et al (2021), Farias et al (2016), Assis et al (2019), Antonio et al (2020), Silva et al (2016), Pires Filho et al (2018), Dias e Gonçalves (2021), Costa et al (2020) e Guia et al (2016) citam a menopausa precoce apenas para relacioná-la à osteoporose como um grande fator de risco, o que condiz com princípios básicos do tratamento de reposição hormonal que tem como uma das funções prevenir a descalcificação acelerada principalmente de mulheres que tiveram a menopausa precoce.

\footnotetext{
${ }^{1}$ Repositório internacional de currículos.
} 
Os artigos citados nesse parágrafo citam a menopausa precoce em artigos com ênfase no fenômeno da menopausa, sendo a precoce um contexto específico dentro do interesse global. Fernandes da Silva et al (2020) dizem, em uma citação da menopausa precoce, que a mesma desencadeia mudanças no ambiente hormonal do corpo da mulher que podem levar a diversas doenças do sistema nervoso central periférico. Dias de Oliveira et al (2021), em pesquisa sobre a possibilidade de usar plantas medicinais para atenuar diferentes sintomatologias do climatério, citam a menopausa precoce como categoria excludente de mulheres da população estudada, assim como Andrade et al (2018) o fazem em estudo sobre a qualidade de vida de mulheres climatéricas atendidas em ambulatório especializado. Melo et al (2017) fazem uma análise sobre a relação entre menopausa e declínio cognitivo, citando a menopausa precoce como uma característica de dispersão sobre o dado "idade" da população analisada. Lima et al (2020), em artigo que aborda os benefícios e riscos atrelados ao tratamento de reposição hormonal pósmenopausa, citam a menopausa precoce ao concluir que

Silveira et al (2020), de forma semelhante a Lima (2020), escrevem artigo sobre o conhecimento clínico sobre a reposição hormonal para mulheres climatéricas em um ambulatório de universidade privada, e citam a menopausa precoce em uma questão de questionário aplicado à população do estudo, para avaliar conhecimento sobre tal, e posteriormente discutem os riscos e benefícios do tratamento de reposição hormonal a partir da bibliografia. Santos et al (2016) publicam artigo sobre a intensidade da sintomatologia climatérica em mulheres pós-menopausa, e citam a menopausa precoce para expor o aumento ao risco de osteoporose, de um lado, e a menopausa precoce e a falta de energia e cansaço crônico, segundo estudo estadunidense sobre o tema. Campos et al (2017) publicam artigo fazendo levantamento de sintomas em mulheres pós-menopausa, e citam a menopausa precoce apenas para caracterizar parte da população do estudo. Buscando analisar terapias alternativas que diminuam os sintomas da menopausa, Araujo et al (2020), citam a menopausa precoce apenas para caracterizá-la dentro do contexto geral da menopausa; da mesma forma o fazem Dorneles et al (2019), ao analisarem o padrão hormonal feminino na menopausa, Correa e Casagrande (2021), ao falarem do papel da melatonina na redução das citocinas IL-6 e IL-17 na menopausa, e Campos Oliveira e Sinico (2020) ao abordarem a alimentação na prevenção e tratamento da osteoporose em mulheres na menopausa.

Por último, há um grupo de publicações com escopos e ênfases tão específicos que não se enquadraram em nenhuma das categorias anteriores nem tiveram coesão interna própria para formarem um conjunto ordenado. Neste grupo estão as publicações de Leite (2020), em artigo sobre saúde cardíaca para mulheres em que cita a menopausa precoce como fator de risco para o coração, Feitosa et al (2017), em publicação sobre o exame colpocitológico em idosas caracterizam a menopausa precoce, apenas, Billerbeck et al (2019) que descrevem a relação entre atividades físicas e tabagismo, abordando a menopausa precoce como efeito do consumo de cigarros, o trabalho de Dechechi Dias et al (2016) sobre alterações nos tecidos nasais em decorrência da menopausa citam a menopausa precoce também na única finalidade de caracterizá-la, e Alencar et al (2020) que escrevem artigo sobre alopecia frontal fibrosante, abordando a menopausa precoce como parte do quadro de uma paciente mas não citando nada além disso.

\section{Conclusão}

Conclui-se que a menopausa precoce pode parecer um termo relativo extensamente citado, com razoável presença dentro da bibliografia e, principalmente, tendo muitos pesquisadores em contato com o conceito. Entretanto, o que a pesquisa quantitativa demonstrou é que de 2016-2021 a menopausa precoce, na verdade, não foi centro de nenhum artigo científico, aparecendo como condição para definir amostra estatística para pesquisas, contextualização para o fenômeno global da menopausa, consequência de patologias como o câncer ou causadora de outros como a osteoporose.

Há uma exceção quando o texto discute os tratamentos de reposição hormonal, onde ganha destaque dada a importância desse tipo de tratamento especificamente no que se refere às mulheres que tenham entrado na menopausa precocemente. Apesar disso este tema ainda não teve uma relevância apontada nos artigos identificou-se uma condição, apesar da importância do tema, 
da falta de interesse das pesquisas no sentido de elevar o debate à esfera pública e mostrar indicadores de melhora da qualidade de vida de mulheres.

Portanto, diversas outras questões presentes como possibilidades de estudos mais robustos e, possivelmente, interdisciplinares sobre a menopausa precoce e as pesquisas feitas nas diferentes regiões no Brasil por pesquisadores homens e mulheres refletem a escassez do campo de pesquisa em saúde deste tema. Faz-se necessário que criem-se políticas públicas mais ativas para restabelecer o debate.

\section{Referências}

Alencar, I. M., Rocha, N. L. Q., Santana, T. S., Marinho, A. L. P., Rabelo, K. P. S., Cardoso, D. C., Ferreira, A. F. P. \& Jucá, N. B. H. (2020) Alopecia frontal fibrosante: relato de caso. Guarujá: Saúde Em Foco: Temas Contemporâneos, 3, 49-55.

Alves, B. W. S., Pereira, F. A., Silva, R. B. B., Alves, J. S., Cruz \& A. R. C. (2021). Avaliação dos fatores de risco para fatura osteoporótica em mulheres pós menopausa. Vargem Grande Paulista: Research, Society and Development, 10(6), 1-7.

Andrade, R. L., Fernandes, A. C. M.; Dias, J. R. P., Laurindo, B. M. \& Vieira, R. C. (2019). Avaliação da qualidade de vida de mulheres climatéricas atendidas em ambulatório especializado. Curitiba: Brazilian Journal of Health Review, 2(1).

Antonio, D. H., Palma, R., Silva, A. P. R., Prestes, S. C. C., Sigolo, J. R. \& Freitas, T. R. O. (2020). O kinect como tratamento na osteoporose pediátrica. Guarujá: Desenvolvimento da Criança e do Adolescente: Evidências Científicas e Considerações Teóricas-Práticas, 3, 679-691.

Antunes, S., Marcelino, O. \& Aguiar, T. (2003). Fisiopatologia da Menopausa. Lisboa: Revista Portuguesa de Clínica Geral, $19(4), 353-357$.

Araujo, A. R., Chagas, R. K. F. \& Lima, I. C. S. (2020). Terapias alternativas para os cuidados dos sintomas da menopausa: delineando possibilidades e desafios. Rio de Janeiro: Revista Online Cuidado é Fundamental, jan./dez., 1267-1273.

Assis, M. E., Calvo, K. \& Maciel, S. S. S. V. (2019). Prevalência de osteoporose e seus fatores de risco associados em mulheres com idade avançada. Belo Horizonte: Revista Acadêmica, 7(1), 109-120.

Barros de Oliveira, D. G., Lopes, H. N., Alves, R. M., Viana, R. S. \& Mathias, C. M. C. (2021). Recuperando a Qualidade de Vida Após o Câncer Feminino. Salvador: Revista Científica, 5(1), 3-9.

Billerbeck, N. C., Borges, L. P., Silva, M. E. A. \& Lima, G. B. (2019). Nível de atividade física e tabagismo. Anápolis: International Journal of Movement Science and Rehabilitation, 1(1), 24-32.

Brischiliari, S. C. R., Dell'Agnolo, C. M., Gil, L. M., Romeiro, T. C., Gravena, A. A. F., De Barros Carvalho, M. D. \& Pelloso, S. M. (2012). Papanicolaou na pós-menopausa: Fatores associados a sua não realização. Rio de Janeiro: Cadernos de Saúde Pública, 28(10), 1976-1984.

Campos, A. Y. S., Fernandes, F. \& Mendonça, C. S. L. (2017). Principais sintomas apresentados por mulheres na pós- menopausa a partir da aplicação do "Índice Menopausal Kupperman e Blatt" e adaptação do "Questionário da Saúde da Mulher". Araçatuba: Anais de publicação das apresentações de Trabalhos de Conclusão de Curso.

Campos Oliveira, J. L. \& Sinico, M. C. (2020). Alimentação na prevenção e tratamento da osteoporose em mulheres na menopausa. Mogi-Guaçu: Interciência \& Sociedade, 5(2), 524-538.

Carvalho, A. M., Carvalho, A. M., Linard, C. F. B. M. \& Carneiro, C. (2020). Osteoporose - uma doença a ser prevenida em todas as fases da vida. Vargem Grande Paulista: Research, Society and Development, 9(10), 1-19.

Correa, R. F. \& Casagrande, T. A. C. (2021). O papel da melatonina na redução das citocinas IL-6 e IL-17 na menopausa. Vargem Grande Paulista: Research, Society and Development, 10(12), 1-11.

Corrêa, C. S. L., Guerra, M. R., Andrade, A. P. S., Carvalho, S. M., Borges, R. M. \& Leite, I. C. G. (2017). Qualidade de vida e fatores associados em mulheres sobreviventes ao câncer do colo do útero. Juiz de Fora: HU Revista, 43(3), 307-315.

Costa, A. M. O., Paz, B. A. \& Almeida, M. A. R. (2020). Avaliação do conhecimento sobre a osteoporose entre estudantes de graduação da área da saúde. Valparaíso de Goiás: Reicen: Revista de Iniciação Científica e Extensão, 3(1), 341-349.

Dechechi Dias, M., Panucci, M. F. S., Kamiguchi, T. M., Telles, T. F. \& Sampaio, C. P. P. (2016). Alterações nasais em mulheres no período do climatério. São Paulo: Arquivos Médicos dos Hospitais e da Faculdade de Ciências Médicas da Santa Casa de São Paulo, 61, 23-27.

Dias, D. F. \& Gonçalves, S. J. C. (2021). Falhas em implantes de quadril. Vargem Grande Paulista: Research, Society and Development, 10(11), 1-11.

Dias de Oliveira, A. K., Oliveira, K. K. D., Souza, L. B. \& Lins, R. H. P. (2021). Uso de plantas medicinais e fitoterápicos no climatério e menopausa. Vargem Grande Paulista: Research, Society and Development, 10(10), 1-18.

Dorneles, A., Fontana, J. \& Zimmermann, C. (2019). Padrão hormonal feminino na menopausa: parâmetros laboratoriais e consequências inestéticas. Santo Ângelo: Revista Saúde Integrada, 12(24).

Farias, L. T. M., Lago, C. C. L. \& Andrade, J. C. S. (2016). Osteoporose: uma análise fisiopatológica voltada para os profissionais da enfermagem. Salvador: Revista Enfermagem Contemporânea, 4(2), 222-236. 
Feitosa, L. M. H., Formiga, L. M. F., Pereira, F. G. F., Araújo, A. K. S., Brandão, A. C. C. \& Rodrigues, A. S. (2017). Realização do colpocitológico em idosas. Recife: Revista de Enfermagem (UFPE Online), 11(9), 3321-3329.

Fernandes da Silva, B., Maciel, C. S., Souza, L. M. M. \& Duarte, L. A. (2020). Menopausa em tempos de COVID-19. Colatina: UNESC em Revista, Edição Especial Covid/pandemia, 2, 213-224.

Fidelis, M., Müller, L. C., Lourenço, E. L. B., Jacomassi, E. \& Boleta-Ceranto, D. C. F. (2020). Perspectiva de tratamento da osteoporose dos maxilares por meio de fitoterápicos: uma revisão da literatura. Cascavel: Revista Thêma et Scientia, 10(2), 118-128.

Guia, C. M., Guia, L. C. M. \& Biondi, R. S. (2016). Tratamento de osteoporose em dois hospitais gerais públicos do perfil de pacientes atendidos em serviços de referência para Distrito Federal, Brasil. Brasília: Brasília MED, 53, 1-5.

Lay, A. A. R. (2018) Idade da menopausa em mulheres idosas do município de São Paulo: fatores associados e análise de sobrevida. São Paulo: Faculdade de Saúde Pública - Universidade de São Paulo.

Leite, C. D. F. C. (2020). Saúde cardiovascular das mulheres do século XXI. Curitiba: Brazilian Journal of Development, 6(11), 90673-90687.

Lima, J. V. O., Cavalcante, L. C. S., Lima, D. C. S., Barbosa, M. C. N. A., Silva, I. P., Luz, A. L. R. \& Costa, M. H. A. (2020). Risco-benefício da terapia de reposição hormonal em mulheres na menopausa. Vargem Grande Paulista: Research, Society and Development, 9(5).

Lovison, K., Taglieti, M., Tomadon, A., Medeiro, K. C., Busatta, B. B. \& Tori, F. S. (2019). Incontinência urinária em mulheres com câncer de mama em tratamento quimioterápico. Toledo: FAG Journal of Health, 1(3), 169-175.

Mairink, A. P. A. R., Gradim, C. V. C., Gozzo, T. O., Canete, A. C. S., Fendrich, L. \& Panobianco, M. S. (2020). A prática sexual de mulheres jovens em tratamento para o câncer de mama. Sorocaba: Escola Ana Nery, 24(3), 1-9.

Melo, C. S. B., Souza, T. S., Teodoro, L. I., Legramanti, S., Fanton, S. V. \& Rückl, S. (2017). Declínio cognitivo e perimenopausa: revisão sistemática. São Paulo: Reprodução e Climatério, 32(2), 132-137.

Oliveira, C. R. C.; Guimarães, M. C. S. \& Machado, R. (2012). Doenças raras como categoria de classificação emergente: o caso brasileiro. Curitiba: DataGramaZero - Revista de Informação, 13(1), 1-10.

Pereira, H. F. B. E. S. A., Viapiana, P. S. \& Silva, K. L. T. (2017). Aspectos Clínicos e Patológicos do Câncer de Mama em Mulheres Jovens Atendidas na FCecon entre 2003 e 2013. Revista Brasileira de Cancerologia, 63(2), 103-109.

Pinho, M. A. T. (2018). Custo-efetividade da vacina HPV quadrivalente, teste HPV e citologia em meio líquido: revisão integrativa. Fortaleza: Universidade Federal do Ceará

Pires Filho, H. S., Uliana Filho, I. I., Perim, L. V. \& Guerra, H. S. (2018). Análise de prontuários de mulheres na pós- menopausa quanto a investigação de osteoporose. Anápolis: Revista Educação em Saúde, 6(2), 49-55.

Prado Filho, F. C. R. C., Andrade, J. M., Silva, C. L., Reis, F. J. C., Nogueira, A. A., Sala, M. M. \& Bighetti, S. (1999). Tumores Pélvicos em Mulheres na Pósmenopausa. São Paulo: Revista Brasileira de Ginecologia e Obstetrícia, 21(1), 47-54.

Santos, T. R., Pereira, S. V. M. \& Lopes Santos, R. (2016) Intensidade da sintomatologia climatérica em mulheres pós-menopausa. Fortaleza: Revista Rene, $17(2), 225-232$

Silva, C. F.F., Amorim, P. R.S., Carvalho, C. J., Sales, S. S. \& Lima, L. M. (2016). Determinantes da densidade mineral óssea na pós-menopausa. Ribeirão Preto: Medicina (Ribeirão Preto Online), 49(1), 26-34.

Silva Oliveira, V. A., Vilarinho, M. L. C. M. \& Milanez, L. S. (2018). Caracterização de mulheres com risco do câncer de mama na Atenção Primária à Saúde. Teresina: Revista de Enfermagem da UFPI, 7(1), 38-43.

Silveira, A. L. R., Rocha, A. C. C., Valentim, G. F. S. \& Marinho, R. M. (2020) Avaliação do grau de conhecimento acerca da terapia de reposição hormonal no climatério em mulheres atendidas no ambulatório de uma faculdade privada em Minas Gerais. Belo Horizonte: Revista Interdisciplinar Ciências Médicas, 4(2).

Sousa, M. T., Silva, M. D. \& Carvalho, R. Revisão integrativa: o que é e como fazer. (2010). Einstein, 8(1).

Vasconcelos, M. R., Farias, N. S., Aoyama, E. A. \& Souza, R. A. G. (2020). Câncer no colo uterino na menopausa em mulheres acima de 45 anos. Revista Brasileira Interdisciplinar de Saúde, 2(1), 88-94.

Wemelinger, M., Machado, M. H., Tavares, M. F. L., Oliveira, E. S., Moyses, N. M. N. \& Ferraz, W. (2010). A feminilização da força de trabalho em saúde no Brasil. Rio de Janeiro: Divulgação em Saúde para o Debate, 45. 